Subscriber access provided by Caltech Library

\title{
Communication
}

\section{Spin Wave Radiation by a Topological Charge Dipole}

Sebastián Alejandro Díaz Santiago, Tomoki Hirosawa, Daniel Loss, and Christina Psaroudaki

Nano Lett., Just Accepted Manuscript • DOI: 10.1021/acs.nanolett.0c02192 • Publication Date (Web): 19 Aug 2020

Downloaded from pubs.acs.org on August 19, 2020

\section{Just Accepted}

"Just Accepted" manuscripts have been peer-reviewed and accepted for publication. They are posted online prior to technical editing, formatting for publication and author proofing. The American Chemical Society provides "Just Accepted" as a service to the research community to expedite the dissemination of scientific material as soon as possible after acceptance. "Just Accepted" manuscripts appear in full in PDF format accompanied by an HTML abstract. "Just Accepted" manuscripts have been fully peer reviewed, but should not be considered the official version of record. They are citable by the Digital Object Identifier (DOI®). "Just Accepted" is an optional service offered to authors. Therefore, the "Just Accepted" Web site may not include all articles that will be published in the journal. After a manuscript is technically edited and formatted, it will be removed from the "Just Accepted" Web site and published as an ASAP article. Note that technical editing may introduce minor changes to the manuscript text and/or graphics which could affect content, and all legal disclaimers and ethical guidelines that apply to the journal pertain. ACS cannot be held responsible for errors or consequences arising from the use of information contained in these "Just Accepted" manuscripts. 


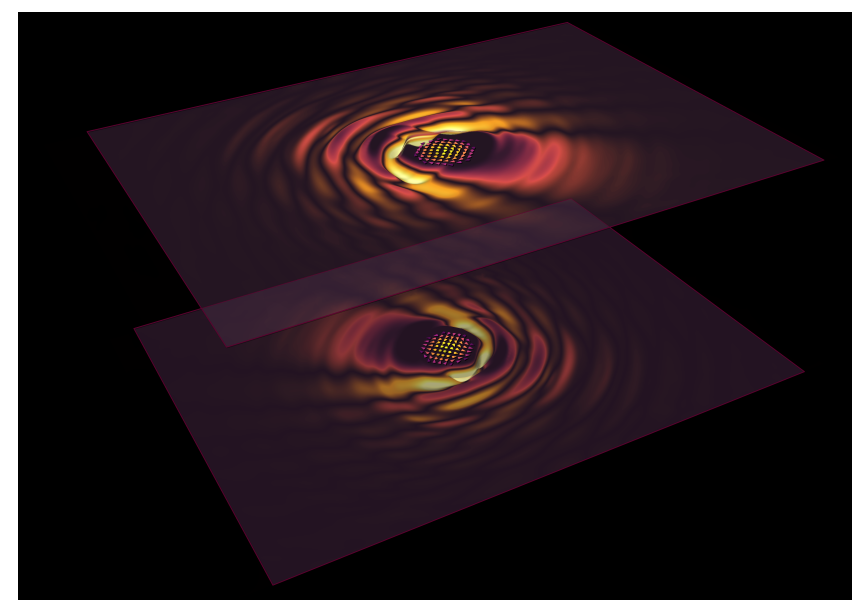

\title{
Spin Wave Radiation by a Topological Charge Dipole
}

\author{
Sebastián A. Díaz, ${ }^{*, \dagger, \|}$ Tomoki Hirosawa, ${ }^{\ddagger}, \|$ Daniel Loss, ${ }^{\dagger}$ and Christina \\ Psaroudaki*,,$\S$ \\ †Department of Physics, University of Basel, Klingelbergstrasse 82, CH-4056 Basel, \\ Switzerland \\ $\ddagger$ Department of Physics, University of Tokyo, Bunkyo, Tokyo 113-0033, Japan \\ \Department of Physics, California Institute of Technology, Pasadena, CA 91125, USA \\ $\S$ Institute for Theoretical Physics, University of Cologne, D-50937 Cologne, Germany \\ $\|$ Contributed equally to this work \\ E-mail: s.diaz@unibas.ch; cpsaroud@caltech.edu
}

\section{Abstract}

The use of spin waves (SWs) as data carriers in spintronic and magnonic logic devices offers operation at low power consumption, free of Joule heating. Nevertheless, the controlled emission and propagation of SWs in magnetic materials remains a significant challenge. Here, we propose that skyrmion-antiskyrmion bilayers form topological charge dipoles and act as efficient sub-100 nm SW emitters when excited by in-plane ac magnetic fields. The propagating SWs have a preferred radiation direction, with clear dipole signatures in their radiation pattern, suggesting that the bilayer forms a SW antenna. Bilayers with the same topological charge radiate SWs with spiral and antispiral spatial profiles, enlarging the class of SW patterns. We demonstrate that the characteristics of the emitted SWs are linked to the topology of the source, allowing for full control of the SW features, including their amplitude, preferred direction of propagation, and wavelength.

\section{Keywords}

magnetic skyrmions, magnonics, spin waves, directional emission, nanoantenna, spintronics

Magnetic skyrmions, particle-like textures in quasi-two-dimensional (2D) systems, are promising elements in future magnetic memory devices, ${ }^{1}$ with complex dynamics governed by topology. ${ }^{2}$ In systems that lack inversion symmetry, isotropic Dzyaloshinskii-Moriya (DM) interactions energetically stabilize skyrmions, ${ }^{3}$ while anisotropic DM interactions can stabilize antiskyrmions ${ }^{4,5}$ with opposite topological charge. ${ }^{6-8}$ The topology of these structures gives rise to fascinating properties, including the topological Hall effect in charge transport $^{9-11}$ and the skyrmion Hall effect, ${ }^{12,13}$ in which skyrmions are deflected in a direc- 


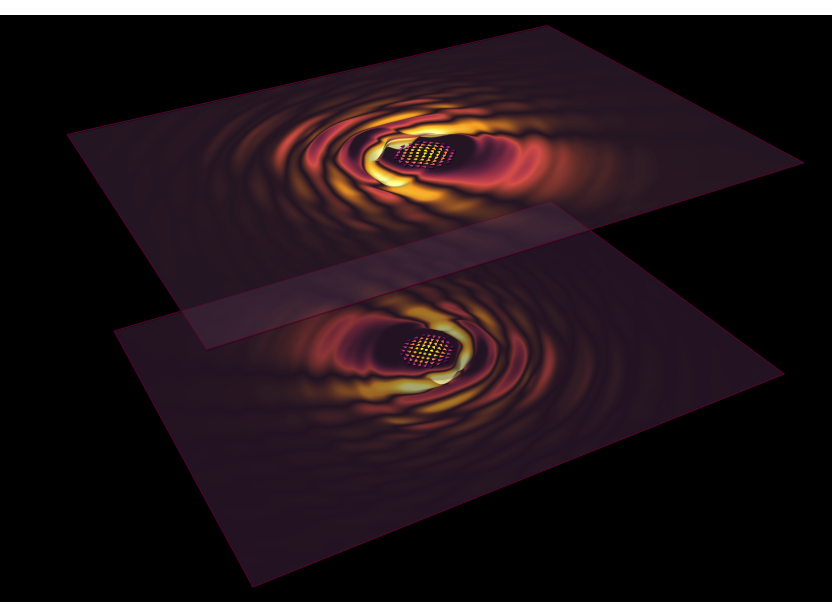

Figure 1: Skyrmion-antiskyrmion bilayer in the presence of an in-plane microwave magnetic field (not shown). The emitted spin wave pattern has dipole signatures, suggesting that the bilayer forms a topological charge dipole that acts as an efficient spin-wave antenna.

tion transverse to the applied force. Skyrmions and antiskyrmions have opposite lateral deviations, ${ }^{14,15}$ and when realized in bilayers lead to the absence of the skyrmion Hall effect. ${ }^{16-18}$

The non-trivial skyrmion topology affects the properties of the surrounding magnons, ${ }^{19,20}$ collective spin excitations, relevant for future magnetic logic and memory devices ${ }^{21,22}$ with tailored properties. ${ }^{23-25} \mathrm{SW}$ currents can be used to transport and process information, free of Joule heating, a significant drawback of modern electronics. Controlled SW emission with nanoscale wavelength, required for the fabrication of miniature devices, has been the subject of intense scientific investigations. ${ }^{26-30} \mathrm{No}-$ tably, noncollinear spin structures, including skyrmions, allow for a controlled SW transmission, ${ }^{31}$ while skyrmion-hosting magnetic insulators are ideal materials for microwave technologies. ${ }^{32}$

Here, by means of micromagnetic simulations, we introduce the concept of a topological charge dipole realized in a skyrmion-antiskyrmion bilayer. An in-plane ac magnetic field activates a counterclockwise (clockwise) rotation of the skyrmion (antiskyrmion) core. Spin waves emitted by the interacting gyrating cores have a preferred propagating direction, with dipole signatures in their radiation pattern, suggest- ing that the bilayer acts as a spin-wave antenna (see Fig. 1). The far-field SW amplitude can be controlled by the interlayer coupling, and the corresponding wavelength is in the sub-100 nm regime. Spiral and antispiral SW patterns are obtained in bilayers with the same topological charge and different helicity, with a rotation that depends on the topology of the source. Skyrmion-antiskyrmion bilayers excited by ac magnetic fields form topological charge dipoles, which besides being of fundamental interest, can be used as efficient SW emitters with enriched and controlled characteristics.

\section{Results}

Skyrmions in Bilayers. The basis for the realization of a topological charge dipole is a bilayer of magnetic materials, such as the one illustrated in Fig. 1. Each layer is described by the model

$$
H=-\frac{1}{2} \sum_{\mathbf{r}, i}\left(J \mathbf{S}_{\mathbf{r}} \cdot \mathbf{S}_{\mathbf{r} \pm a \mathbf{e}_{i}}+D \mathbf{d}_{ \pm \mathbf{e}_{i}} \cdot \mathbf{S}_{\mathbf{r}} \times \mathbf{S}_{\mathbf{r} \pm a \mathbf{e}_{i}}\right)+H_{z},
$$

where $H_{z}=-\sum_{\mathbf{r}} g \mu_{B} B S_{\mathbf{r}}^{z}$ is the Zeeman energy. $J$ and $D$ represent the exchange and DM couplings respectively. The two layers are coupled through a ferromagnetic interaction, $H_{\text {int }}=-J_{\text {int }} \sum_{\mathbf{r}} \mathbf{S}_{\mathbf{r}}^{1} \cdot \mathbf{S}_{\mathbf{r}}^{2}$, with $J_{\text {int }}>0$, the interlayer coupling. $J_{\text {int }}$ can be tuned experimentally by introducing a spacer between the two layers. ${ }^{33}$ The magnetization dynamics is governed by the total Hamiltonian, $H=H_{1}+H_{2}+H_{\text {int }}+$ $H_{\mathrm{osc}}$, with $H_{\mathrm{osc}}=-\sum_{\mathbf{r}} g \mu_{B} B_{0} \cos (\omega t)\left(n_{\mathbf{r}}^{x, 1}+\right.$ $\left.n_{\mathbf{r}}^{x, 2}\right)$ describing the presence of a time-periodic in-plane magnetic field of frequency $\omega$, and is calculated by a numerical solution of the Landau-Lifshitz-Gilbert (LLG) equation (see the Supporting Information (SI) for details).

The model of Eq. (1) supports magnetization textures characterized by an integer topological charge

$$
Q=\frac{1}{4 \pi} \int d \mathbf{r} \mathbf{n} \cdot\left(\partial_{x} \mathbf{n} \times \partial_{y} \mathbf{n}\right),
$$

defined as a measure of the wrapping of the 
magnetization $\mathbf{n}(\mathbf{r})$ around a unit sphere, and the helicity $\gamma$. Both the sign and value of $Q$ and $\gamma$ are determined by the orientation of the DM vector $\mathbf{d}_{ \pm \mathbf{e}_{i}}$, which is dictated by the crystal symmetry of a given material. We consider four cases of crystal symmetry related to skyrmionhosting materials, namely the cubic symmetry of noncentrosymmetric materials, ${ }^{34,35}$ the interfacial symmetry of magnetic thin films on nonmagnetic metals, ${ }^{36}$ the $C_{2 v}$ symmetry of a double layer Fe on W(110), ${ }^{4}$ and the $D_{2 d}$ symmetry of acentric tetragonal Heusler compounds. ${ }^{5}$ The various types of DM vectors and the corresponding magnetization textures are summarized in Fig. 2-(a)-(b).

We envision a bilayer as a stack of two different materials each hosting a texture that could be either a skyrmion or an antiskyrmion (Bloch or Néel). The various composite pairs are summarized in Table 1, and are categorized into three different types. Type I includes all possible combinations of particles with opposite $Q$, Type II of the same $Q$ and $\gamma$, and Type III of the same $Q$ but different $\gamma$. As we demonstrate below, the dynamics of the topological particles, as well as the characteristics of the emitted SWs, strongly depend on the composite pair type. In the presence of a finite interlayer coupling $J_{\text {int }}$, the two particles interact via a potential of the form $E_{\text {int }}\left(R_{0}\right)=$ $J_{\text {int }} \int d \mathbf{r}\left[1-\mathbf{n}_{1}\left(\mathbf{r}-\mathbf{R}_{1}\right) \cdot \mathbf{n}_{2}\left(\mathbf{r}-\mathbf{R}_{2}\right)\right] d \mathbf{r},{ }^{33}$ where $\mathbf{R}_{i}$ are the collective coordinates of position for each particle, ${ }^{37,38}$ and $R_{0}=\left|\mathbf{R}_{1}-\mathbf{R}_{2}\right| . E_{\text {int }}\left(R_{0}\right)$ is depicted in Fig. S6, and has a strong dependence on both $Q$ and $\gamma$.

The onset of an in-plane oscillating magnetic field activates the counterclockwise (CCW) rotation of the skyrmion core, which has been experimentally measured in the skyrmion-hosting ferrimagnetic insulator $\mathrm{Cu}_{2} \mathrm{OSeO}_{3}$ in the $\mathrm{GHz}$ regime. ${ }^{39,40}$ This localized skyrmion deformation has energy $\mathcal{E}_{0} \approx \varepsilon_{\text {gap }}$, where $\varepsilon_{\text {gap }}=g \mu_{B} B$ is the gap of the propagating spin waves with energy $\mathcal{E}=\varepsilon_{\text {gap }}+\left(\hbar^{2} / 2 m\right) k^{2}$, where $k$ is the radial momentum and $m=\hbar^{2} / 2 J S^{2} a^{2}$ is the magnon mass. To discover the role of $Q$ and $\gamma$ on magnons, we numerically diagonalize the spin wave Hamiltonian, provided in Supplementary Note 5 in the SI. Although the magnon spectrum is found to be insensitive to the choice of $Q$ and $\gamma$, we found that local modes of the skyrmion with a CCW sense of gyration, correspond to clockwise (CW) modes for the antiskyrmion. We numerically confirm the existence of a CCW (CW) mode for the skyrmion (antiskyrmion) with energy $\mathcal{E}_{0} \approx \varepsilon_{\text {gap }}$. These modes describe a rotation of the out-of-plane spin components around the (anti)skyrmion core in a $(\mathrm{CW}) \mathrm{CCW}$ manner. The same results are recovered within the continuum model, given in Supplementary Note 6 in the SI.

Topological Charge Dipole as a SW antenna. To analyze the characteristics of the emitted SWs we focus on two salient quantities; the particle's center of mass $\mathbf{R}_{i}$ and the amplitude of the far-field emitted SWs. The former corresponds to the collective coordinates of position of the skyrmion core at each layer $i=1,2,{ }^{41}$

$$
R_{i}^{\nu}=\frac{1}{Q_{i}} \int d \mathbf{r} r^{\nu} \mathbf{n}_{i} \cdot\left(\partial_{x} \mathbf{n}_{i} \times \partial_{y} \mathbf{n}_{i}\right)
$$

written here for the continuum model, with $\nu=x, y$, while an expression for the discrete model is given in Eq. (S3). We numerically verify that $H_{\mathrm{osc}}$ activates the CCW (CW) mode for a skyrmion (antiskyrmion), signaled by a resonance peak at $\omega \simeq b$, with $b=g \mu_{B} B / J S$ the uniform magnetic field in dimensionless units. For $J_{\text {int }}=0, \omega=0.6=b$, each of the uncoupled layers acts as a monopole and emits radially symmetric SWs. The emitted SWs correspond to fluctuations of the $z$-component of the magnetization above the oscillating ferromagnetic background, $\delta n_{\mathbf{r}}^{z}(t)=n_{\mathbf{r}}^{z}(t)-n_{\mathrm{FM}}^{z}(t)$. Unless explicitly stated, we study $\delta n_{\mathbf{r}}^{z, 1}$ of layer 1 , while emitted spin waves in layer 2 have a similar structure. In particular, we find $\delta n_{\mathbf{r}}^{z, 2} \simeq \delta n_{\mathbf{r}}^{z, 1}$ for Type II and III, and $\delta n_{\mathbf{r}}^{z, 2} \simeq \delta n_{-\mathbf{r}}^{z, 1}$ for Type I, with $\mathbf{r} \gg \lambda$, where $\lambda$ is the skyrmion radius. Thus, the excited SWs represent collective modes of the bilayer. Snapshots of $\delta n_{\mathbf{r}}^{z}(t)$ for all Types of bilayers are depicted in Fig. 2(c)-(e) (yellow panels), while the full time evolution of the SW pattern is visualized in Supplementary Movies 1, 4, and 5 for Types I-(a), 
(a)

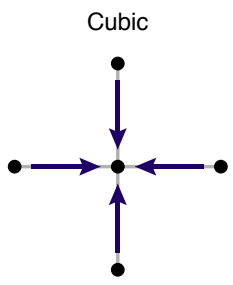

(b) Skyrmion Bloch (SB)

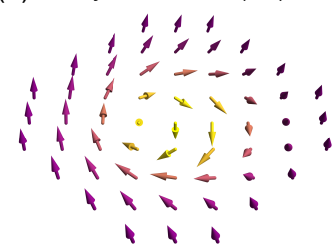

$Q=-1, \gamma=-\frac{\pi}{2}$

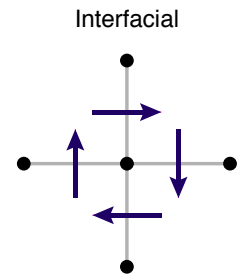

Skyrmion Néel (SN)

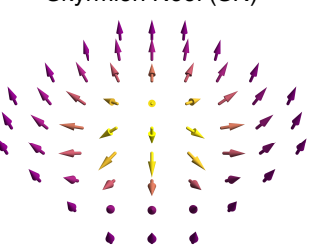

$Q=-1, \gamma=0$

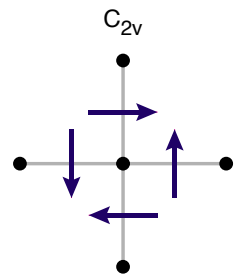

Antiskyrmion Néel (AN)

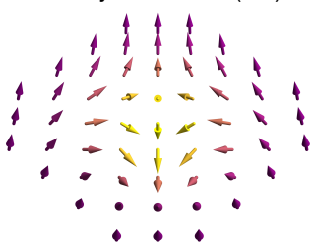

$Q=1, \gamma=\pi$

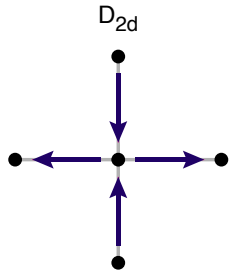

Antiskyrmion Bloch (AB)

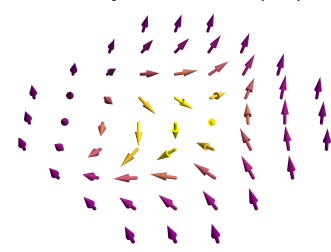

$Q=1, \gamma=\frac{\pi}{2}$

(c) Type I

(d)

(e)

Type III
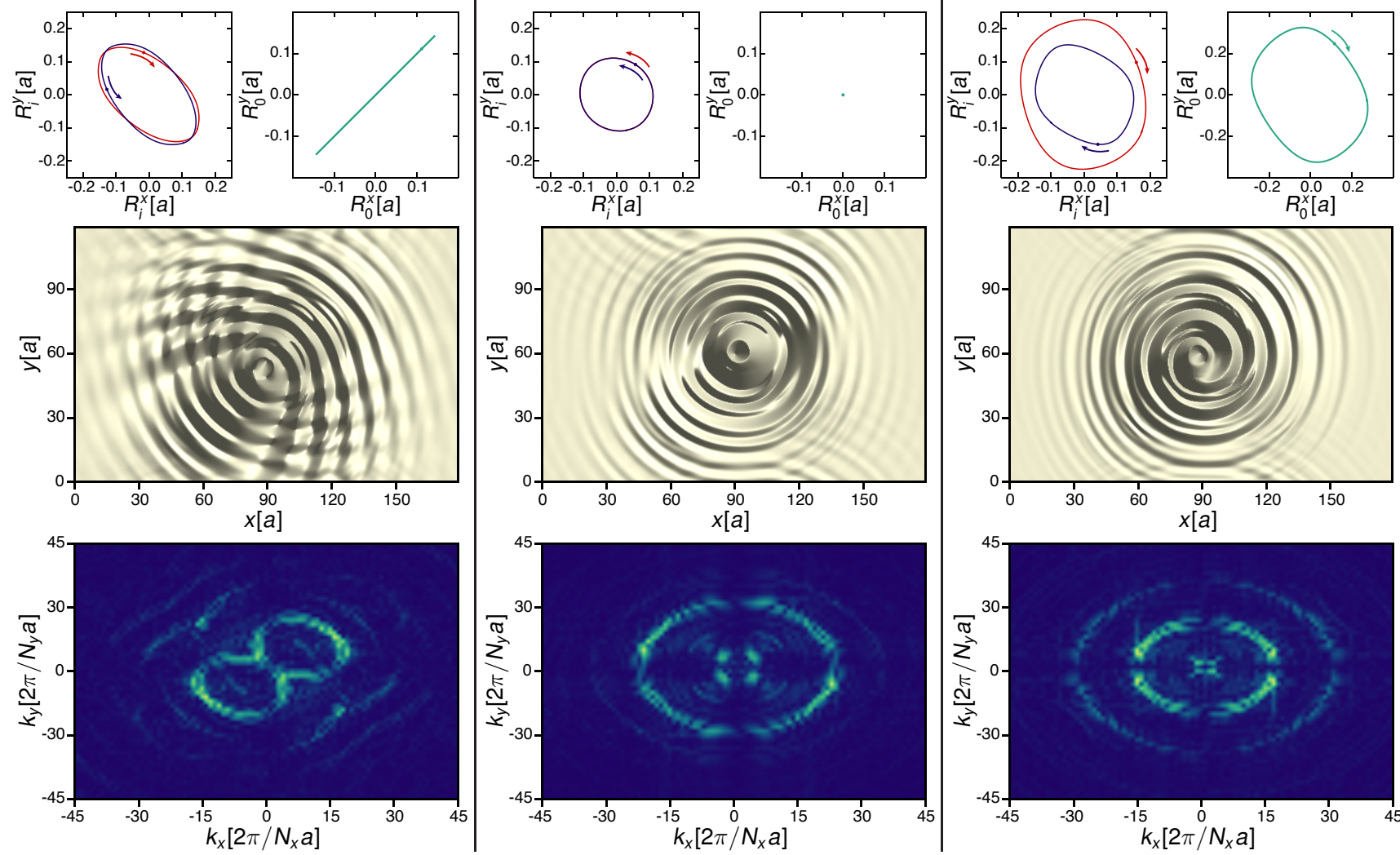

Figure 2: (a)-(b) Relation between crystal symmetry and spin configuration. (a) Sketch of the DM vectors (blue arrows) between n.n. sites of the lattice for cubic, interfacial, $C_{2 v}$ and $D_{2 d}$ symmetry. (b) Spin field configuration, corresponding to different DM interactions. In all cases, the $z$-component of magnetization is aligned parallel to the external out-of-plane magnetic field. A Bloch skyrmion (SB) with $\gamma=-\pi / 2$ and $Q=-1$, a Néel skyrmion (SN) with $\gamma=0$ and $Q=-1$, a Néel antiskyrmion (AN) with $\gamma=\pi$ and $Q=1$, and a Bloch antiskyrmion (AB) with $\gamma=\pi / 2$ and $Q=1$. (c)-(e) Relation between the dynamics of the collective coordinate of position $\mathbf{R}_{i}(t)$ and the features of the radiated SWs. In all cases we use $J / D=1, b=0.6\left(B=0.6 J S / g \mu_{B}\right)$, and $\omega=0.6(\tilde{\omega}=0.6 J S / \hbar)$. Blue lines denote the path of $\mathbf{R}_{i}$ for the texture in layer 1 , and red lines for layer 2, while arrows indicate the sense of gyration. The green lines denote the path of the charge separation distance $\mathbf{R}_{0}=\mathbf{R}_{1}-\mathbf{R}_{2}$. Yellow panels depict snapshots of the perpendicular magnetization deviation $\delta n_{\mathbf{r}}^{z}(t)$, while the radiation pattern $\delta n_{\mathbf{k}}^{z}$ is illustrated in the blue panels. 
Table 1: Composite pairs of skyrmions and antiskyrmions. Type I includes all possible combinations of particles with opposite topological charge, Type II of the same charge and helicity, and Type III of the same charge but different helicity. We introduce $\tilde{Q}=Q_{1}+Q_{2}$, and $\tilde{\gamma}=\gamma_{1}-\gamma_{2}$.

\begin{tabular}{c|c|c|c|c||c|c|c|c||c|c}
\hline & \multicolumn{9}{|c|}{ Type I } & \multicolumn{1}{c}{ Type II } \\
\hline Subtype & a & b & c & d & a & b & c & d & a & b \\
Composite Pair & SB-AN & SN-AB & SN-AN & SB-AB & SB-SB & SN-SN & AN-AN & AB-AB & SB-SN & AN-AB \\
$(\tilde{Q}, \tilde{\gamma})$ & $(0,-3 \pi / 2)$ & $(0,3 \pi / 2)$ & $(0,-\pi)$ & $(0,-\pi)$ & $(-2,0)$ & $(-2,0)$ & $(2,0)$ & $(2,0)$ & $(-2,-\pi / 2)$ & $(2,3 \pi / 2)$ \\
\hline
\end{tabular}

II-(a), and III-(a) respectively. Our simulations clearly show that, collective SW modes are generated by the gyrating interacting topological charges, and propagate from the source to the edge of the sample with characteristics related to the bilayer Type. Type I emits directional, Type II symmetrical, and Type III spiral SWs.

Fig. 2-(c)-(e) summarizes the relation between the dynamics of $\mathbf{R}_{i}(t)$ and the features of the radiated SWs, for all three Types, for $J_{\text {int }}=0.3 J$, and the resonance condition $\omega=$ $0.6=b$. For Type I both the skyrmion (blue line) and the antiskyrmion (red line) follow elliptical paths, with opposite sense of gyration, while the charge separation distance $\mathbf{R}_{0}=\mathbf{R}_{1}-\mathbf{R}_{2}$, pointing from the negative to the positive charge, oscillates back and forth along a straight line (green line). This is understood by employing Thiele's approach to obtain the equation of motion $-4 \pi Q \epsilon_{\nu \mu} \dot{R}_{0}^{\mu}=$ $F_{\nu} \cos (\omega t)$, where $F_{x}=c \sin (\tilde{\gamma} / 2)$, and $F_{y}=$ $c \cos (\tilde{\gamma} / 2)$, allowing for a dependence on the helicity difference $\tilde{\gamma}=\gamma_{1}-\gamma_{2}$ (see Supplementary Note 6 in the SI for a discussion on the Thiele equation). Provided that $R_{0}^{y}(t) / R_{0}^{x}(t)=$ $-F_{x} / F_{y}$, the charge separation distance performs a time-periodic motion on a straight path obtained by rotating the $x$ axis by an angle $\psi=\tan ^{-1}[\sin (\tilde{\gamma})]$, illustrated in Fig. S4-(a)-(c) for the various subtypes with different $\tilde{\gamma}$.

The second prominent feature when examing Fig. 2 is the directionality of the radiated SWs. The behavior of Fig. 2-(c) (yellow panel), where we depict a snapshot of $\delta n_{\mathbf{r}}^{z}(t)$ for the Type I-(a) bilayer, implies that a topological charge dipole creates directional spin waves, explored further in Fig. S4-(d)-(f) for the various subtypes. The preferred radiation direction coincides with the direction of $\mathbf{R}_{0}$ at an angle $\psi=\tan ^{-1}[\sin (\tilde{\gamma})]$. This is supported by the radiation pattern $\delta n_{\mathbf{k}}^{z}$ illustrated in Fig. 2- (c) (blue panel), which presents the characteristic two source dipole feature, suggesting that $\delta n_{\mathbf{r}}^{z}(t)$ has an azimuthal $\phi$ distribution of the form $\sim \sin (\phi-\psi)^{27,42,43}$ [see Supplementary Note 3 in the SI for a definition of $\left.\delta n_{\mathbf{k}}^{z}\right]$. The helicity $\tilde{\gamma}$ of the charge dipole can be used to manipulate the propagation direction of the spin waves in the 2D plane, as illustrated in Fig. S4 and Supplementary Movies 1, 2, and 3, respectively.

In Fig. 2, further results are provided for the SW generation process in Type II and III. In Type II, both textures with the same $Q$ and $\gamma$ perform identical paths, with $\mathbf{R}_{0}=0$. SWs exhibit symmetric radiation patterns [see Fig. 2(d)], as the result of a single monopole emitter. In Type III bilayers, consisting of textures with the same $Q$ and different $\gamma$, a Néel texture performs a larger circular path (red line) compared to a Bloch texture (blue line), while $\mathbf{R}_{0}$ follows a circular path (green line). A fascinating feature is the creation of spiral outwards travelling SWs [see Fig. 2-(e) yellow panel for Type III(a)]. The physics of the spiral-shape formation is understood in terms of the gyrating motion of $\mathbf{R}_{0}$, which plays the role of the source for magnetization oscillations. ${ }^{44,45}$ The direction of the spiral rotation, as illustrated in Fig. S1, depends on $Q$. Spirals of Type III-(a), with $\mathbf{R}_{0}$ circulating in a CCW manner, have opposite direction of rotation compared to antispirals of Type III-(b), with $\mathbf{R}_{0}$ circulating in a CW manner.

Far-Field Spin Wave Amplitude. We now focus on the characteristics of the on-resonance directional SWs of Type I bilayers, and identify the signatures of the charge interaction in the radiation field. To study the features of the emitted SWs irrespective of a particular direc- 

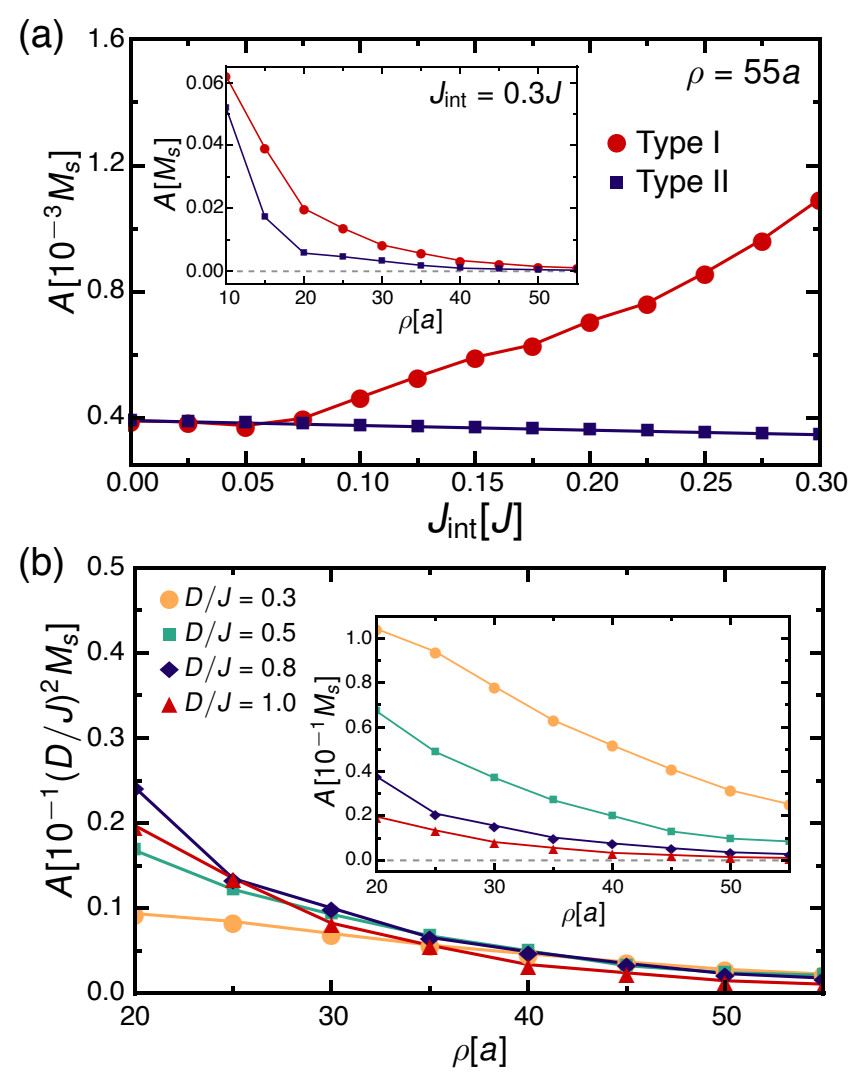

Figure 3: (a) Far-field amplitude $A$ as a function of interlayer coupling $J_{\text {int }}$ for $\rho=55 a$, $b=0.6\left(B=0.6 J S / g \mu_{B}\right)$, and $\omega=0.6(\tilde{\omega}=$ $0.6 J S / \hbar)$. A increases linearly with $J_{\text {int }}$ for Type I bilayers (red line) and shows a weak dependence for Type II (blue line). The inset depicts the dependence of $A$ on $\rho$ for $J_{\text {int }}=0.3 J$, and as expected vanishes fast due to the finite Gilbert damping $\alpha$. (b) Far-field amplitude $A$ of Type I-(a), as a function of $\rho$, for $J_{\text {int }}=0.3 J, b=0.6\left(B=0.6 J S / g \mu_{B}\right), \omega=0.6$ $(\tilde{\omega}=0.6 J S / \hbar)$, and four values of the ratio $D / J$. $A$ is scaled with $(D / J)^{2}$ such that the values for different ratios converge for distances sufficiently away from the source, $\rho \gtrsim 30 a$.

tion, we define the far-field SW amplitude as

$$
A=\frac{M_{s}}{N_{\mathcal{J}}} \sum_{\mathbf{r} \in \mathcal{J}} \frac{1}{2}\left(\max _{t \in[0, T]}\left[\delta n_{\mathbf{r}}^{z}(t)\right]-\min _{t \in[0, T]}\left[\delta n_{\mathbf{r}}^{z}(t)\right]\right),
$$

over the area $\mathcal{J}$ of an annulus centered around the skyrmion core, with inner (outer) radius $\rho$ $(P)$, comprised of $N_{\mathcal{J}}$ lattice sites. $T=2 \pi / \omega$ is the period of the ac field, and we use $P=\rho+5 a$ throughout. In the limit $\rho \rightarrow 55 a$, thus at the edge of the simulated area, $A \rightarrow 0$ due to the finite Gilbert damping $\alpha$.

Fig. 3-(a) summarizes the dependence of $A$ on $J_{\text {int }}$ for the Type I (II) bilayer illustrated by the red (blue) line. The linear dependence of $A$ on $J_{\text {int }}$ for the Type I bilayer indicates that the source of the directional SW radiation is indeed an interacting charge dipole, and that changing $J_{\text {int }}$ is an excellent mechanism to tune the SW amplitude. For Type II, $A$ shows a weak dependence on $J_{\text {int }}$, suggesting that even when the layers interact, the SWs remain unaffected. The inset of Fig. 3 shows the dependence of $A$ on $\rho$, and as expected, vanishes fast due to the finite $\alpha$. Finally, Type III shows an irregular behavior on $J_{\text {int }}$ presented in Fig. S2.

The far-field amplitude $A$ can be enhanced by tuning the $D / J$ ratio, presented in Fig. 3(b), for four different values of $D / J$. We use $J_{\text {int }} / J=\{0.3,0.2,0.05,0.012\}$, for $D / J=$ $\{1,0.8,0.5,0.3\}$ to realize textures of approximately the same size and avoid deformations of the skyrmion profile. We observe that $A$ is increased as $D / J$ is decreased with a $(J / D)^{2}$ scaling. Finally, we reveal an additional mechanism to tune $A$ by varying the out-of-plane magnetic field $b$, presented in Fig. S3. By increasing $b$, $A$ is decreased fast, while for $b \gtrsim 0.7$ all local perturbations of the $z$-component vanish. We expect that $A$ can be further manipulated by tuning the layer thickness ${ }^{46}$ and the perpendicular magnetic anisotropy, ${ }^{17}$ an investigation that we leave for future work. The choice of $\alpha=0.03, J / D=5$, and $\rho=30 a$, results in an amplitude of about $15-20 \% \times M_{s}$.

Dispersion Characterization. An estimate of the dispersion relation of the emitted SWs is obtained by analyzing the radiation patterns $\delta n_{\mathbf{k}}^{z}$ for the various bilayer Types as a function of the excitation frequency $\omega$. We examine the results of Fig 4-(a), where we plot the radiation pattern $\delta n_{\mathbf{k}}^{z}$, for $J_{\text {int }}=0.3 J, b=0.6$, and two frequencies, the resonance frequency $\omega=\omega_{\text {res }}$, and $\omega=0.7$. For $\omega=\omega_{\text {res }}$ there is a distinct short wavelength propagating mode at $k_{\text {res }}=0.65 a^{-1}$ for Type I, $k_{\text {res }}=0.8 a^{-1}$ for Type II, and $k_{\text {res }}=0.57 a^{-1}$ for Type III, with $k^{2}=k_{x}^{2}+k_{y}^{2}$ the radial momentum, indicated 
with a red rhombus on the colored surface of $\delta n_{\mathbf{k}}^{z}$ of Fig. 4 -(a). The corresponding intensity $\delta n_{k_{\text {res }}}^{z}$, plotted in Fig. 4-(b), takes its maximum at $\omega_{\text {res }}$ and decreases fast as $\omega$ increases. A small shift of the resonance frequency is observed for Type I, $\omega_{\text {res }}=0.63$, probably caused by a shift of the CCW (CW) energy at finite $J_{\text {int }}$. We conclude that $k_{\text {res }}$ is a short wavelength resonant mode activated by the gyrotropic motion of the particle core, further enhanced by the bilayer coupling and with properties linked to the topology of the emitter.

Besides the resonant mode, we identify a long wavelength mode with a $k$ vector indicated by a yellow circle in Fig. 4-(a). This is the usual SW mode expected for the ferromagnetic layer, with a quadratic dispersion $\mathcal{E} / J S=b+a^{2} k^{2}$, plotted in Fig. 4-(c). For frequencies away from resonance, the amplitude of this mode dominates over the magnon spectrum, while its wavelength can be tuned by varying the external frequency $\omega$. To reach however, the wavelength of the resonant mode at $k_{\text {res }}=0.63 a^{-1}$ and $\omega=0.6$, a larger excitation frequency $\omega=1$ is required. In the inset of Fig. 4-(c), we depict small deviations of the recorded energy values $\mathcal{E}$ from the quadratic dispersion at small $k$ wavevectors. Whether such deviations are the result of the finite system size considered here, deserves further future investigation. In physical units of $J=1 \mathrm{meV}, J / D=4, J_{\text {int }} / J=0.3$, $B=324 \mathrm{mT}, a=1 \mathrm{~nm}, S=1$ and excitation frequency $\omega=57 \mathrm{GHz}$, a skyrmion of radius $44 \mathrm{~nm}$ emits resonance modes of wavelength $\lambda=2 \pi J a / D k_{\text {res }}=42 \mathrm{~nm}$. Due to the nanoscale of the skyrmion core, the emitted SWs have sufficiently short wavelengths in the sub-100 nm regime.

\section{Discussion}

We theoretically studied the formation of topological charge dipoles in skyrmion-antiskyrmion bilayers, with a directional SW radiation that exhibits clear dipole signatures in the radiation pattern. The topological charge separation performs a time-periodic motion along the direction of the SW radiation, determined by
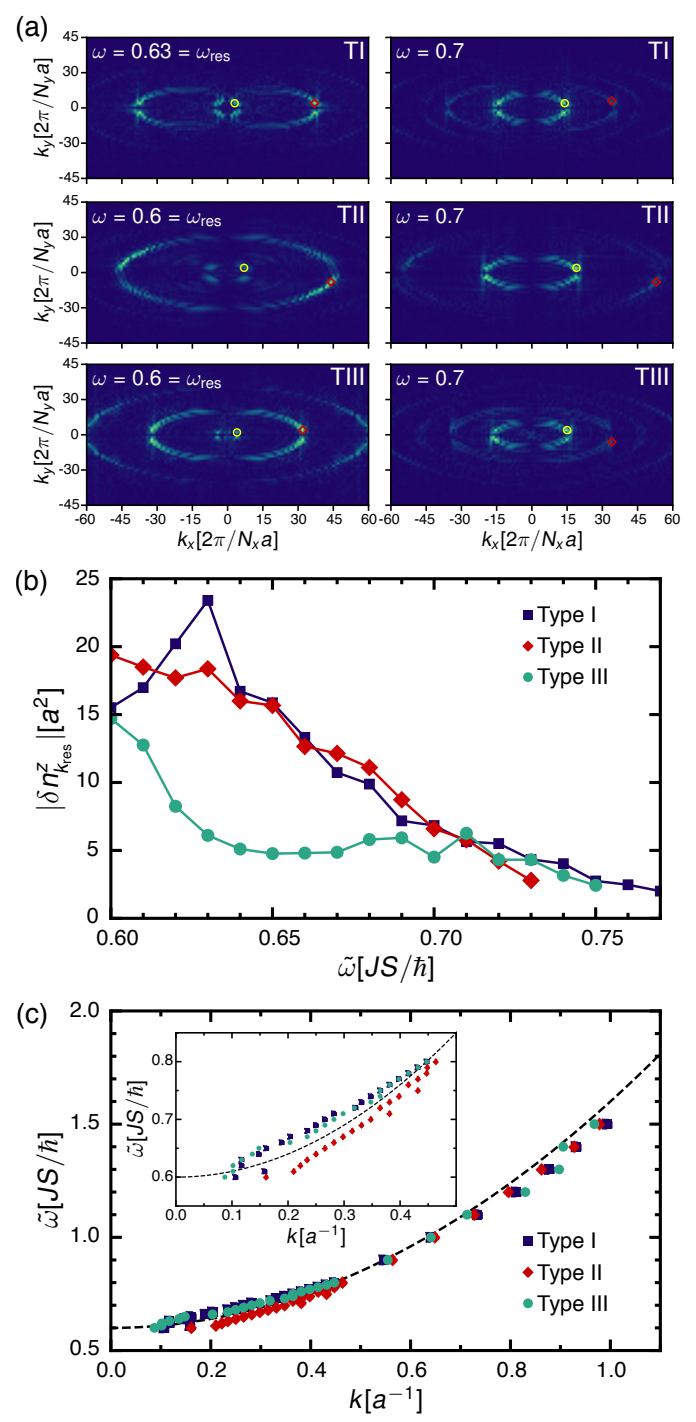

Figure 4: (a) Radiation patterns $\delta n_{\mathbf{k}}^{z}$ for all Types of bilayers, for $J_{\mathrm{int}}=0.3 J, b=0.6(B=$ $\left.0.6 J S / g \mu_{B}\right)$, and two frequencies, $\omega=\omega_{\text {res }}$ and $\omega=0.7$. The location of the resonant mode $k_{\text {res }}$ is indicated by a red rhombus, while the wavevector of the ferromagnetic SW mode is indicated by a yellow circle. (b) The amplitude of the $k_{\text {res }}$ mode, $\left|\delta n_{k_{\text {res }}}^{z}\right|$, as a function of the excitation frequency $\tilde{\omega}$, given here in physical units $\tilde{\omega}=\omega J S / \hbar$. This is a resonant mode with an intensity that vanishes for $\omega>\omega_{\text {res }}$. The resonance frequency is $\omega_{\text {res }}=0.6$ for Types II and III, and slightly shifted to $\omega_{\text {res }}=0.63$ for Type I. (c) Dispersion of the ferromagnetic SWs, for all three Types of bilayers. The dashed line indicates the theoretically predicted quadratic relation $\mathcal{E} / J S=b+a^{2} k^{2}$. The inset depicts small deviations of the recorded energy values from the quadratic dispersion at small $k$ wavevectors. 
the helicity of the source. The magnetic dipole fields are collective modes of the bilayer with sufficiently short wavelengths in the sub-100 $\mathrm{nm}$ regime, and a far-field amplitude controlled, among other mechanisms, by the interlayer coupling. SWs with a spiral or antispiral pattern can emerge in bilayers hosting particles with the same $Q$ but different $\gamma$, with a sense of rotation that depends on the sign of the topological charge $Q$.

To realize the topological charge dipole experimentally in a stack of two materials separated by a spacer, a confining geometry such as a nanodisk, ${ }^{47}$ can be utilized to ensure that the skyrmion and antiskyrmion are approximately on top of each other. Alternatively, isolated skyrmions can be generated in each layer using electric currents, ${ }^{48}$ or at the local edge of a magnetic layer under certain magnetic field protocols. ${ }^{49}$ For a precise positioning, skyrmions can be driven towards engineered pinning defects.

Efficient spin wave emission has been the topic of intensive theoretical and experimental investigations. Spin torque nano-oscillators $(\mathrm{STNO})^{50}$ can be used to create directional spin wave radiation, ${ }^{27}$ as the result of interference patterns of excitations originating from two or more STNO placed in an array. Spin waves with a spiral structure are emitted from gyrating dynamical skyrmions in Spin-Hall oscillators, ${ }^{44}$ and have been experimentally observed in a stack of dynamically excited vortex cores. ${ }^{26,51}$ In our proposed system, the SW generation mechanism is reliable and robust as it does not rely on spin wave interference, it can be realized in a large variety of skyrmion-hosting materials, including magnetic insulators, and is independent of a particular fabrication of magnetic elements with tailored properties. ${ }^{52,53}$ Besides the fundamental interest of our results, we anticipate that our findings could lead to the development of novel efficient SW emitters and SW antennas, with tunable characteristics linked to the topology of the source.

\section{Supporting Information}

The Supporting Information is available free of charge at...

Details of the micromagnetic simulations, further characteristics of the emitted spin waves, spin wave spectrum on the discrete lattice, bilayer interacting Hamiltonian, simplified Thiele's equation of motion, and supplementary movies of all the bilayer types.

\section{Acknowledgments}

T.H. is grateful to T. Hinokihara for useful discussions. T.H. was supported by the Japan Society for the Promotion of Science through Program for Leading Graduate Schools (MERIT), JSPS KAKENHI (Grant No. 18J21985) and Young Researchers' Exchange Program between Japan and Switzerland 2018. C.P. has received funding from the European Union's Horizon 2020 research and innovation programme under the Marie Sklodowska-Curie grant agreement No 839004. S.A.D. and D.L. were supported by the Swiss National Science Foundation (Switzerland) and the NCCR QSIT.

\section{References}

(1) Fernández-Pacheco, A.; Streubel, R.; Fruchart, O.; Hertel, R.; Fischer, P.; Cowburn, R. P. Three-dimensional nanomagnetism. Nature Communications 2017, 8, $1-14$.

(2) Everschor-Sitte, K.; Masell, J.; Reeve, R. M.; Kläui, M. Perspective: Magnetic skyrmions - Overview of recent progress in an active research field. Journal of Applied Physics 2018, 124, 240901.

(3) Bogdanov, A.; Hubert, A. Thermodynamically stable magnetic vortex states in magnetic crystals. Journal of Magnetism and Magnetic Materials 1994, 138, 255 269. 
(4) Hoffmann, M.; Zimmermann, B.; Müller, G. P.; Schürhoff, D.; Kiselev, N. S.; Melcher, C.; Blügel, S. Antiskyrmions stabilized at interfaces by anisotropic Dzyaloshinskii-Moriya interactions. Nature Communications 2017, 8, 308.

(5) Nayak, A. K.; Kumar, V.; Ma, T.; Werner, P.; Pippel, E.; Sahoo, R.; Damay, F.; Rößler, U. K.; Felser, C.; Parkin, S. S. P. Magnetic antiskyrmions above room temperature in tetragonal Heusler materials. Nature 2017, 548, 561566.

(6) Koshibae, W.; Nagaosa, N. Theory of antiskyrmions in magnets. Nature Communications 2016, 7, 10542.

(7) Camosi, L.; Rougemaille, N.; Fruchart, O.; Vogel, J.; Rohart, S. Micromagnetics of antiskyrmions in ultrathin films. Phys. Rev. B 2018, 97, 134404.

(8) Güngördü, U.; Nepal, R.; Tretiakov, O. A.; Belashchenko, K.; Kovalev, A. A. Stability of skyrmion lattices and symmetries of quasi-two-dimensional chiral magnets. Phys. Rev. B 2016, 93, 064428.

(9) Neubauer, A.; Pfleiderer, C.; Binz, B.; Rosch, A.; Ritz, R.; Niklowitz, P. G.; Böni, P. Topological Hall Effect in the $A$ Phase of MnSi. Phys. Rev. Lett. 2009, 102, 186602.

(10) Lee, M.; Kang, W.; Onose, Y.; Tokura, Y.; Ong, N. P. Unusual Hall Effect Anomaly in MnSi under Pressure. Phys. Rev. Lett. 2009, 102, 186601.

(11) Schulz, T.; Ritz, R.; Bauer, A.; Halder, M.; Wagner, M.; Franz, C.; Pfleiderer, C.; Everschor, K.; Garst, M.; Rosch, A. Emergent electrodynamics of skyrmions in a chiral magnet. Nature Physics 2012, 8, 301-304.

(12) Litzius, K.; Lemesh, I.; Krüger, B.; Bassirian, P.; Caretta, L.; Richter, K.;
Büttner, F.; Sato, K.; Tretiakov, O. A.; Förster, J.; Reeve, R. M.; Weigand, M.; Bykova, I.; Stoll, H.; Schütz, G.; Beach, G. S. D.; Kläui, M. Skyrmion Hall effect revealed by direct time-resolved X-ray microscopy. Nature Physics 2017, 13, 170175.

(13) Jiang, W.; Zhang, X.; Yu, G.; Zhang, W.; Wang, X.; Benjamin Jungfleisch, M.; Pearson, J. E.; Cheng, X.; Heinonen, O.; Wang, K. L.; Zhou, Y.; Hoffmann, A.; Velthuis, S. G. E. Direct observation of the skyrmion Hall effect. Nature Physics 2017, 13, 162-169.

(14) Leonov, A. O.; Mostovoy, M. Edge states and skyrmion dynamics in nanostripes of frustrated magnets. Nature Communications 2017, 8, 14394.

(15) Everschor-Sitte, K.; Sitte, M.; Valet, T.; Abanov, A.; Sinova, J. Skyrmion production on demand by homogeneous DC currents. New Journal of Physics 2017, 19, 092001.

(16) Huang, S.; Zhou, C.; Chen, G.; Shen, H.; Schmid, A. K.; Liu, K.; Wu, Y. Stabilization and current-induced motion of antiskyrmion in the presence of anisotropic Dzyaloshinskii-Moriya interaction. Phys. Rev. B 2017, 96, 144412.

(17) Zhang, X.; Zhou, Y.; Ezawa, M. Magnetic bilayer-skyrmions without skyrmion Hall effect. Nature Communications 2016, 7 , 10293.

(18) Zhang, X.; Ezawa, M.; Zhou, Y. Thermally stable magnetic skyrmions in multilayer synthetic antiferromagnetic racetracks. Phys. Rev. B 2016, 94, 064406.

(19) Díaz, S. A.; Klinovaja, J.; Loss, D. Topological Magnons and Edge States in Antiferromagnetic Skyrmion Crystals. Phys. Rev. Lett. 2019, 122, 187203.

(20) Díaz, S. A.; Hirosawa, T.; Klinovaja, J.; Loss, D. Chiral magnonic edge states 
in ferromagnetic skyrmion crystals controlled by magnetic fields. Phys. Rev. Research 2020, 2, 013231.

(21) Khitun, A.; Bao, M.; Wang, K. L. Magnonic logic circuits. Journal of Physics D: Applied Physics 2010, 43, 264005.

(22) Gertz, F.; Kozhevnikov, A.; Filimonov, Y.; Khitun, A. Magnonic Holographic Memory. IEEE Transactions on Magnetics 2015, 51, 1-5.

(23) Lenk, B.; Ulrichs, H.; Garbs, F.; Münzenberg, M. The building blocks of magnonics. Physics Reports 2011, 50\%, $107-136$.

(24) Serga, A. A.; Chumak, A. V.; Hillebrands, B. YIG magnonics. Journal of Physics D: Applied Physics 2010, 43, 264002.

(25) Yu, H.; d' Allivy Kelly, O.; Cros, V.; Bernard, R.; Bortolotti, P.; Anane, A.; Brandl, F.; Heimbach, F.; Grundler, D. Approaching soft X-ray wavelengths in nanomagnet-based microwave technology. Nature Communications 2016, 7, 11255.

(26) Wintz, S.; Tiberkevich, V.; Weigand, M.; Raabe, J.; Lindner, J.; Erbe, A.; Slavin, A.; Fassbender, J. Magnetic vortex cores as tunable spin-wave emitters. $\mathrm{Na}$ ture Nanotechnology 2016, 11, 948-953.

(27) Macià, F.; Hoppensteadt, F. C.; Kent, A. D. Spin wave excitation patterns generated by spin torque oscillators. Nanotechnology 2014, 25, 045303.

(28) Demidov, V. E.; Kostylev, M. P.; Rott, K.; Münchenberger, J.; Reiss, G.; Demokritov, S. O. Excitation of short-wavelength spin waves in magnonic waveguides. $A p$ plied Physics Letters 2011, 99, 082507.

(29) Tsoi, M.; Jansen, A. G. M.; Bass, J.; Chiang, W.-C.; Tsoi, V.; Wyder, P. Generation and detection of phase-coherent current-driven magnons in magnetic multilayers. Nature 2000, 406, 46-48.
(30) Chang, L.-J.; Chen, J.; Qu, D.; Tsai, L.Z.; Liu, Y.-F.; Kao, M.-Y.; Liang, J.-Z.; Wu, T.-S.; Chuang, T.-M.; Yu, H.; Lee, S.F. Spin Wave Injection and Propagation in a Magnetic Nanochannel from a Vortex Core. Nano Letters 2020, 20, 3140-3146, PMID: 32323994.

(31) Duerr, G.; Huber, R.; Grundler, D. Enhanced functionality in magnonics by domain walls and inhomogeneous spin configurations. Journal of Physics: Condensed Matter 2011, 24, 024218.

(32) Garst, M.; Waizner, J.; Grundler, D. Collective spin excitations of helices and magnetic skyrmions: review and perspectives of magnonics in non-centrosymmetric magnets. Journal of Physics D: Applied Physics 2017, 50, 293002.

(33) Koshibae, W.; Nagaosa, N. Theory of skyrmions in bilayer systems. Scientific Reports 2017, 7, 42645.

(34) Butenko, A. B.; Leonov, A. A.; Rößler, U. K.; Bogdanov, A. N. Stabilization of skyrmion textures by uniaxial distortions in noncentrosymmetric cubic helimagnets. Phys. Rev. B 2010, 82, 052403 .

(35) Yu, X. Z.; Kanazawa, N.; Onose, Y.; Kimoto, K.; Zhang, W. Z.; Ishiwata, S.; Matsui, Y.; Tokura, Y. Near roomtemperature formation of a skyrmion crystal in thin-films of the helimagnet FeGe. Nature Materials 2011, 10, 106-109.

(36) Heinze, S.; von Bergmann, K.; Menzel, M.; Brede, J.; Kubetzka, A.; Wiesendanger, R.; Bihlmayer, G.; Blügel, S. Spontaneous atomic-scale magnetic skyrmion lattice in two dimensions. Nature Physics 2011, 7, 713-718.

(37) Psaroudaki, C.; Hoffman, S.; Klinovaja, J.; Loss, D. Quantum Dynamics of Skyrmions in Chiral Magnets. Phys. Rev. X 2017, r, 041045. 
(38) Psaroudaki, C.; Loss, D. Skyrmions Driven by Intrinsic Magnons. Phys. Rev. Lett. 2018, 120, 237203.

(39) Onose, Y.; Okamura, Y.; Seki, S.; Ishiwata, S.; Tokura, Y. Observation of Magnetic Excitations of Skyrmion Crystal in a Helimagnetic Insulator $\mathrm{Cu}_{2} \mathrm{OSeO}_{3}$. Phys. Rev. Lett. 2012, 109, 037603.

(40) Okamura, Y.; Kagawa, F.; Mochizuki, M.; Kubota, M.; Seki, S.; Ishiwata, S.; Kawasaki, M.; Onose, Y.; Tokura, Y. Microwave magnetoelectric effect via skyrmion resonance modes in a helimagnetic multiferroic. Nature Communications 2013, 4, 2391.

(41) Papanicolaou, N.; Tomaras, T. Dynamics of magnetic vortices. Nuclear Physics B 1991, 360, $425-462$.

(42) Macià, F.; Kent, A. D.; Hoppensteadt, F. C. Spin-wave interference patterns created by spin-torque nanooscillators for memory and computation. Nanotechnology 2011, 22, 095301.

(43) Laine, S. M.; Thuneberg, E. V. Spin-wave radiation from vortices in ${ }^{3} \mathrm{He}-\mathrm{B}$. Phys. Rev. B 2018, 98, 174516.

(44) Giordano, A.; Verba, R.; Zivieri, R.; Laudani, A.; Puliafito, V.; Gubbiotti, G.; Tomasello, R.; Siracusano, G.; Azzerboni, B.; Carpentieri, M.; Slavin, A.; Finocchio, G. Spin-Hall nano-oscillator with oblique magnetization and Dzyaloshinskii-Moriya interaction as generator of skyrmions and nonreciprocal spin-waves. Scientific Reports 2016, 6, 36020.

(45) Li, L.; Liao, X.; Chan, K. H.; Zhang, K. On nonlinear multiarmed spiral waves in slowly rotating fluid systems. Physics of Fluids 2010, 22, 011701.

(46) Osuna Ruiz, D.; Parra, E. B.; Bukin, N.; Heath, M.; Lara, A.; Aliev, F. G.; Hibbins, A. P.; Ogrin, F. Y. Dynamics of spiral spin waves in magnetic nanopatches:
Influence of thickness and shape. Phys. Rev. B 2019, 100, 214437.

(47) Moreau-Luchaire, C.; Moutafis, C.; Reyren, N.; Sampaio, J.; Vaz, C. A. F.; Van Horne, N.; Bouzehouane, K.; Garcia, K.; Deranlot, C.; Warnicke, P.; Wohlhüter, P.; George, J.-M.; Weigand, M.; Raabe, J.; Cros, V.; Fert, A. Additive interfacial chiral interaction in multilayers for stabilization of small individual skyrmions at room temperature. Nature Nanotechnology 2016, 11, 444-448.

(48) Jiang, W.; Upadhyaya, P.; Zhang, W.; Yu, G.; Jungfleisch, M. B.; Fradin, F. Y.; Pearson, J. E.; Tserkovnyak, Y.; Wang, K. L.; Heinonen, O.; te Velthuis, S. G. E.; Hoffmann, A. Blowing magnetic skyrmion bubbles. Science 2015, 349, 283-286.

(49) Du, H.; Che, R.; Kong, L.; Zhao, X.; Jin, C.; Wang, C.; Yang, J.; Ning, W.; Li, R.; Jin, C.; Chen, X.; Zang, J.; Zhang, Y.; Tian, M. Edge-mediated skyrmion chain and its collective dynamics in a confined geometry. Nature Communications 2015, 6, 8504 .

(50) Demidov, V. E.; Urazhdin, S.; Demokritov, S. O. Direct observation and mapping of spin waves emitted by spin-torque nano-oscillators. Nature Materials 2010, 9, 984-988.

(51) Behncke, C.; Adolff, C. F.; Lenzing, N.; Hänze, M.; Schulte, B.; Weigand, M.; Schütz, G.; Meier, G. Spin-wave interference in magnetic vortex stacks. Communications Physics 2018, 1, 50.

(52) Mamica, S.; Krawczyk, M.; Sokolovskyy, M. L.; Romero-Vivas, J. Large magnonic band gaps and spectra evolution in three-dimensional magnonic crystals based on magnetoferritin nanoparticles. Phys. Rev. B 2012, 86, 144402. 
(53) Sklenar, J.; Bhat, V. S.; Tsai, C. C.; DeLong, L. E.; Ketterson, J. B. Generating wave vector specific Damon-Eshbach spin waves in Py using a diffraction grating. Applied Physics Letters 2012, 101, 052404. 